\title{
On the Opening of the Journal of Astrophysics and Aerospace Technology
}

\section{Shikui Tang*}

Department of Astronomy, University of Massachusetts, Amherst, USA

It is my great pleasure to serve the Editorial Board of Journal of Astrophysics and Aerospace Technology- A new journal which has a tremendous potential to grow in the related fields.

An open access journal is helpful for the growth of the astrophysics and aerospace community, scientifically and educationally. All articles, even including the audio version if available, can be accessible without any limitation. In addition, published papers can be even viewed using the preferred language with the aid of translation tools. These features enable worldwide exposure of any interesting finding quickly. The professional reviewers will make sure the correctness all published articles.

Astrophysics aims to solve the mystery of the universe on the basis of collected evidences. With the rapid development of technology in the recent several decades, tremendous observations have been collecting; many long-lasting problems in astrophysics have been examined and explored. However, the more information we obtain, the more myster- ies we find, which in turn drives us to continue our scientific journey further and deeper. As an editor, I think the key to make our journal successful is to always keep an open mind altitude towards divergent scientific opinions and views, especially on those issues which may not have a clear answer or may have more than one explanation.

Albert Einstein ever said, "The most beautiful thing we can experience is the mysterious. It is the source of all true art and science." For it, I could not agree more. Our journal will always respect diverse opinions on these mysterious as long as they are sustained by arguments. With the first issue published, the Journal of Astrophysics and Aerospace Technology provides a platform for people working in these fields to exchange opinions and enhance communication and cooperation. I believe with the support of our authors, referees, and most importantly, the readers, the Journal of Astrophysics and Aerospace Technology will soon establish its own reputation in the astrophysics community in the near future.
*Corresponding author: Shikui Tang, Research Fellow, Department of Astronomy, University of Massachusetts, Amherst, USA, Tel: 215-615-5627; E-mail: shktang@gmail.com

Received April 11, 2011; Accepted April 12, 2011; Published April 16, 2012

Citation: Tang S (2012) On the Opening of the Journal of Astrophysics and Aerospace Technology. J Astrophys Aerospace Technol 1:e101. doi:10.4172 पिय०स००.1000e101

Copyright: @ 2012 Tang S. This is an open-access article distributed under the terms of the Creative Commons Attribution License, which permits unrestricted use, distribution, and reproduction in any medium, provided the original author and source are credited. 